$$
\begin{aligned}
& \text { AE } \\
& \text { AUG } 20 \text { OgO } \\
& \text { OST TA-A22879 } \\
& \text { CONF-980560-- }
\end{aligned}
$$

\title{
AN EVALUATION OF KINETIC EFFECTS IN THE DIII-D DIVERTOR
}

\author{
by \\ J.G. WATKINS, O. BATISHCHEV, J.A. BOEDO, D.N. HILL, \\ C.J. LASNIER, R. LEHMER, A.W. LEONARD, and R.A. MOYER
}

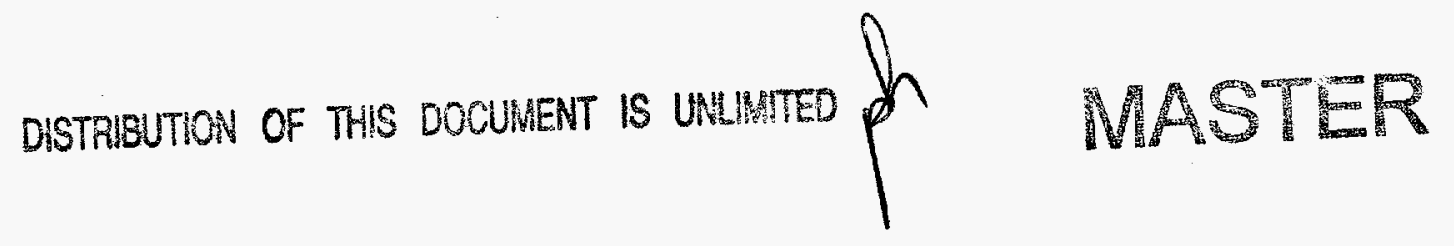




\section{DISCLAIMER}

This report was prepared as an account of work sponsored by an agency of the United States Government. Neither the United States Government nor any agency thereof, nor any of their employees, makes any warranty, express or implied, or assumes any legal liability or responsibility for the accuracy, completeness, or usefulness of any information, apparatus, product, or process disclosed, or represents that its use would not infringe privately owned rights. Reference herein to any specific commercial product, process, or service by trade name, trademark, manufacturer, or otherwise does not necessarily constitute or imply its endorsement, recommendation, or favoring by the United States Government or any agency thereof. The views and opinions of authors expressed herein do not necessarily state or reflect those of the United States Government or any agency thereof. 


\section{DISCLAIMER}

Portions of this document may be illegible electronic image products. Images are produced from the best available original document. 


\title{
AN EVALUATION OF KINETIC EFFECTS IN THE DIII-D DIVERTOR
}

\author{
by \\ J.G. WATKINS, † O. BATISHCHEV, $\ddagger$ J.A. BOEDO, $\diamond$ D.N. HILL, $\triangle$ \\ C.J. LASNIER,,$\Delta$ R. LEHMER, $\diamond$ A.W. LEONARD, and R.A. MOYER $\diamond$
}

This is a preprint of a paper to be presented at the 13th International Conference on Plasma Surface Interactions on Controlled Fusion Devices, May 18-23, 1998, San Diego, California and to be published in Journal of Nuclear Materials.

\author{
tSandia National Laboratories, Albuquerque \\ ‡Lodestar/Massachusetts Institute of Technology \\ $\checkmark$ University of California, San Diego \\ $\Delta$ Lawrence Livermore National Laboratory
}

\begin{abstract}
Work supported by the U.S. Department of Energy under Contracts DE-AC03-89ER51114, DE-AC04-95-AL85000, W-7405-ENG-48, and Grants DE-FG02-97ER54392, DE-FG0291ER54109, DE-FG03-95ER54294
\end{abstract}

GA PROJECT 3466

AUGUST 1998 


\section{ABSTRACT}

Under certain conditions, high temperature electrons diffusing across the separatrix near the midplane can propagate to the divertor without collision and produce a high energy tail in the electron distribution function near the separatrix. We evaluate a kinetic criterion along the field using measurements from divertor Thomson scattering and compare temperature measurements from target plate Langmuir probes to estimate the magnitude of the high energy tail. A FokkerPlanck kinetic model [O. Batishchev, Phys. Plasmas 4, 1672 (1998)], which includes electronelectron, electron-ion, electron-neutral, and charge exchange interactions is used to estimate the size of the high energy tail. For the cases considered, we found less than $1 \%$ of non-thermal particles near the target plate at typical densities and power levels for the DIII-D scrape-off layer plasma. ELMs violate our kinetic criterion. 
2 


\section{INTRODUCTION}

Diverted Tokamaks typically have an electron temperature at the midplane that is several times higher than the divertor target plate. Electrons in the tail of the distribution are less collisional than those in the bulk and are therefore the most likely to reach the target plate without collisions in the divertor plasma. These effects are expected to become important when the temperature scale length along a field line is short compared to the collision length of the tail electrons through the divertor plasma [1]. In the transition to detached divertor plasmas, the density becomes higher which increases collisionality but the temperature scale length becomes shorter and tends to enhance the non-thermal tail. In this paper, we explore these effects in two types of plasma discharges.

Many divertor measurements in tokamaks are done by Langmuir probes which are sensitive to the tail of the distribution function and there is concern that the electron temperature measurements can be in error by a factor of 3 to 5 if the population of fast electrons is high enough [2]. Estimates from Ref. 2 indicate the $T_{e}$ discrepancy would appear for the ELMing $\mathrm{H}$-mode with a midplane to divertor $\mathrm{T}_{\mathrm{e}}$ ratio of 3 if the fast tail particles in the divertor get above $1 \%$ of the background plasma. In DII-D, we are fortunate to have a divertor Thomson scattering system [3] which can measure electron temperature of the bulk of the distribution and provides a method for determining if significant non-Maxwellian electrons are present. 
4 


\section{METHOD}

Plasma conditions in DIII-D can influence the degree to which these kinetic effects are observable. We have chosen two experimental conditions to examine in this paper which we expect would result in very different distribution functions in the divertor plasma. ELMing $\mathrm{H}$-mode with $6.5 \mathrm{MW}$ of neutral beam injection yields an attached hot plasma with higher midplane temperature, higher divertor density due to high recycling, and more power flow to the target plate. By contrast, PDD plasmas typically have a $2 \mathrm{eV}$ electron temperature near the target plate with steeper temperature gradients near the X-point. The partially detached plasmas result in conditions that can produce discrepancies between probes and Thomson scattering [5].

To evaluate the criterion for kinetic effects to become important in each of the above cases, we have used Thomson scattering measurements near and above the target plate which were obtained during ELMing H-mode and PDD plasma discharges. These measurements were mapped to the magnetic geometry using the equilibrium reconstruction solutions and other mapping tools to obtain an average value of the density and temperature profiles along the magnetic field line at various lengths from the target plate. We have averaged our collections of measurements on the flux surface near the separatrix between normalized poloidal flux values of 1.0 and 1.002 (roughly $2 \mathrm{~cm}$ at the target plate). The kinetic criterion is then calculated from the data to estimate the importance of kinetic effects locally along a field line. The criterion is defined as the ratio of the mean-free-path of midplane bulk electrons along the field $(0.2 \times$ $\left.\mathrm{T}_{\mathrm{mid}} 2 / \mathrm{n}_{\text {local }}\right)$ to the electron temperature scale length $\mathrm{T}_{\text {local }} /(\mathrm{dT} / \mathrm{dx})_{\text {local }}$.

Langmuir probe measurements of electron temperature are sensitive to non-Maxwellian electrons and have recently been improved for low $T_{e}$. Probe measurement of $T_{e}$ is performed by sampling the tail of the distribution function. Collisionless electrons from farther away along the magnetic field line are expected to distort the parallel distribution function which is preferentially sampled by the probe. The number of samples in the characteristic just below the floating potential was increased by a factor of 2 by incorporating a special wave form generated from a cubic polynomial. This change also allowed us to increase the sweep frequency from $250 \mathrm{~Hz}$ to $500 \mathrm{~Hz}$ in order to improve the time resolution of the measurements while not violating the current ramp rate limit of our power supply at low temperature and high density.

Details of the fitting technique can affect the results. Using the asymmetric double probe fitting technique [6,7] does lower the resulting temperature but requires measurements at electron saturation which are not normally possible at higher levels of additional heating power ( $>5 \mathrm{MW}$ ) on DIII-D with our present power supplies (4 A limit) and probe geometry. This technique therefore can not presently be employed routinely in fitting DIII-D probe data. 
The divertor Thomson scattering measures the bulk population of electrons. The perpendicular velocity distribution is more accurately characterized by the bulk temperature sampled by the laser while the probe measurements are more characteristic of the parallel velocity distribution. The $30 \mathrm{~ns}$ laser measurements in the divertor occur at $50 \mathrm{~ms}$ intervals $(20 \mathrm{~Hz})$. ELMs and higher frequency fluctuations are present which makes comparison more difficult.

The Fokker-Planck model [8] we use here is a one dimensional in space and 2.5D in velocity. Observations were converted to parallel length along the field line to the target plate for comparison and calculation of the local kinetic criterion. In this paper, we will focus on the $T_{e}$ measurements. 


\section{OBSERVATIONS}

\subsection{ATTACHED ELMING H-MODE PLASMA}

For an attached ELMing H-mode plasma the Thomson scattering system measures that the $\mathrm{T}_{\mathrm{e}}$ drops continuously from about $100 \mathrm{eV}$ at the midplane $(25 \mathrm{~m})$ to about $25-30 \mathrm{eV}$ at the target plate (Fig. 1). The density increases by about the same factor, so the pressure is constant. We see the temperature gradient is somewhat steeper below the $\mathrm{X}$-point (at about $10 \mathrm{~m}$ from the target) and the density gradient increases in this region. The density and temperature points at $5 \mathrm{~m}$ appear to be about $20 \%$ low (for constant pressure) due to our flux surface averaging. We have included the steeper gradients in our calculations to be conservative in the evaluation of the kinetic criterion also shown in Fig. 1. Since the gradients are not steep enough to overcome the higher density, we do not expect strong kinetic effects in this case. From this distribution function (Fig. 2), we calculate the tail population is $0.3 \%$ of the background electron density. We expect the $\mathrm{T}_{\mathrm{e}}$ measurements to agree based on the estimates from Ref. 2.
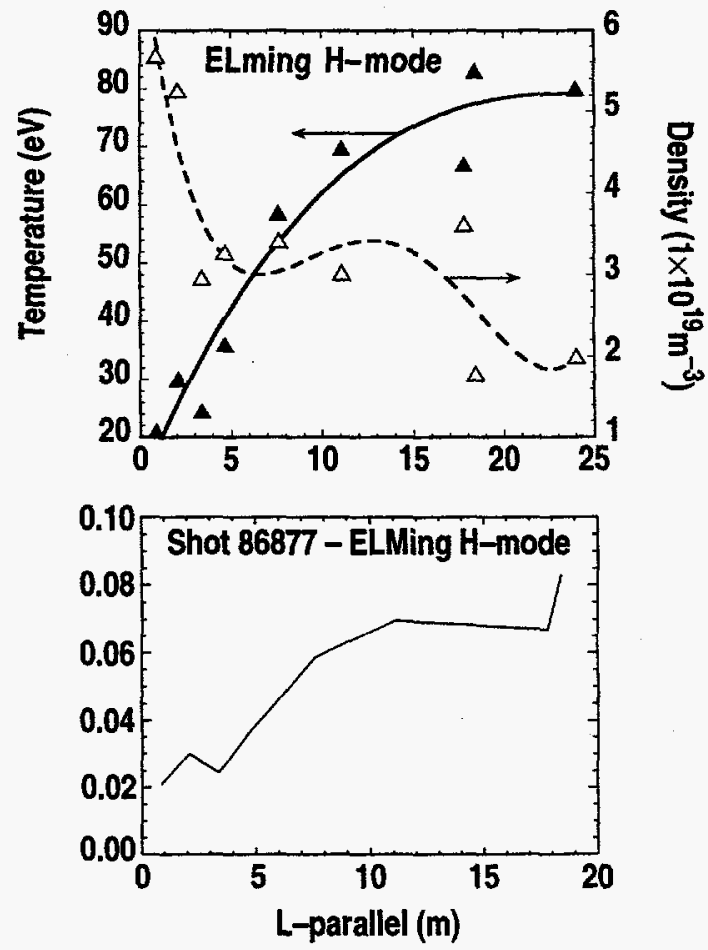

Fig. 1. These conditions near the separatrix $(1<$ normalized poloidal flux $(p s i-n)<1.002)$ along the field during ELMing $H$-mode (a) and the resulting kinetic criterion (b) are based on Thomson scattering data in the divertor. The gradients are not steep enough near the divertor plate to overcome the collisionality at the higher density. 


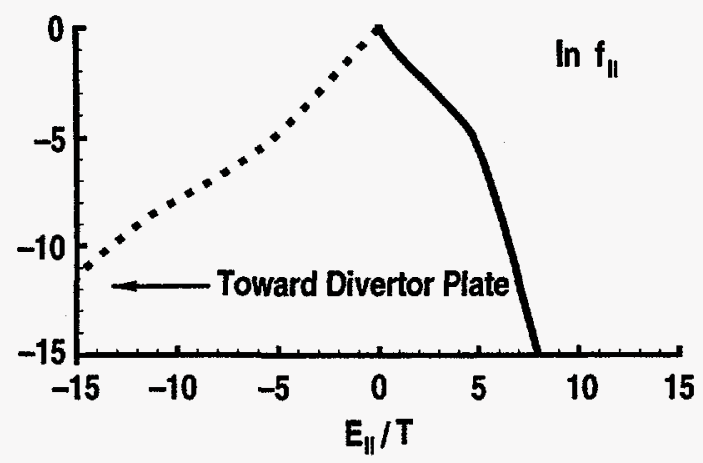

Fig. 2. The distribution function just above the target plate and calculated from the Fokker Planck code for ELMing $H$-mode is shown as a function of the normalized energy distribution of the bulk population of electrons.

The $T_{e}$ measurements from the Thomson scattering and the Langmuir probes do agree as shown in Fig. 3. Here, we have constructed a radial profile of the plasma temperature using the lower Thomson channel and the target plate Langmuir probes as the strike point is moved past the point of measurement. The higher $\mathrm{T}_{e}$ measurements in the outer scrape-off layer are probably associated with turbulence and a low signal/noise ratio which will be discussed in a later section. From our floating potential measurements with the reciprocating probe across the outer divertor leg separatrix, we observe a very narrow strongly negative $V_{f}$ layer near the separatrix $[2,5,10]$ which could indicate the presence of a higher energy tail in this very narrow region. This might not show up on our $\mathrm{T}_{\mathrm{e}}$ comparison but deserves further study.

\subsection{PARTIALLY DETACHED PLASMA}

In a PDD plasma with deuterium puffing, the Thomson measurements mapped along the field line (Fig. 4) show that the $\mathrm{T}_{\mathrm{e}}$ gradient extends further up the field than in the ELMing

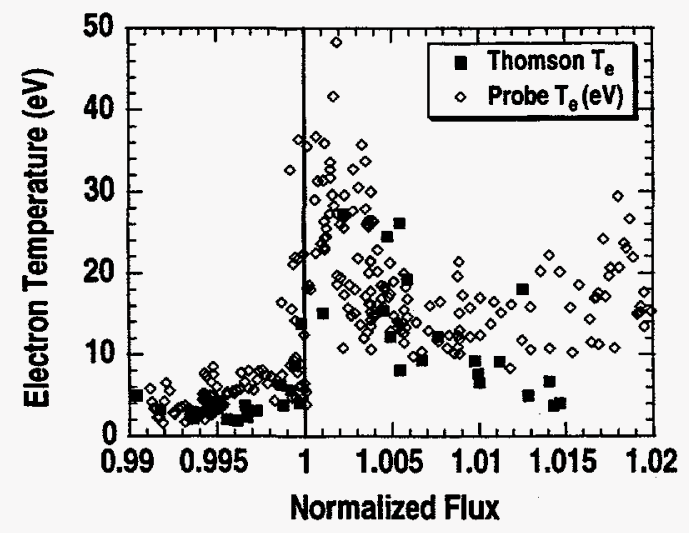

Fig. 3. This figure shows a comparison of the $T_{e}$ profiles in ELMing H-mode. The diamonds are Langmuir probe data and the boxes are measurements of Thomson scattering. 
(a)

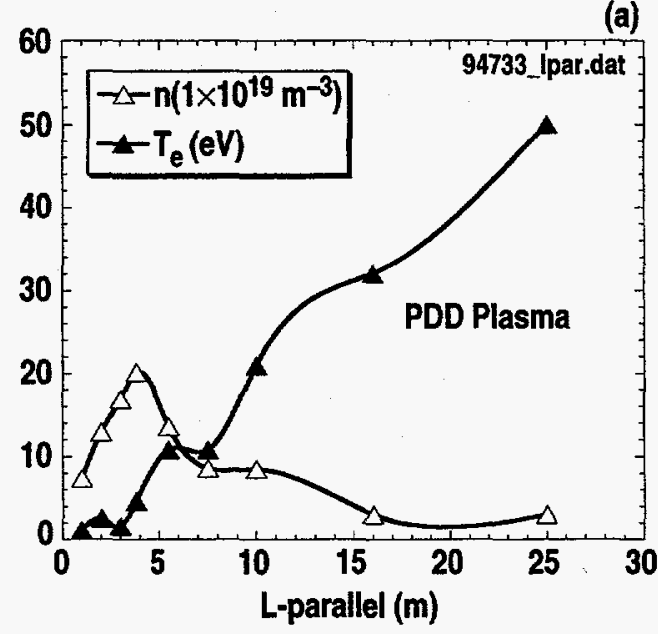

(b)

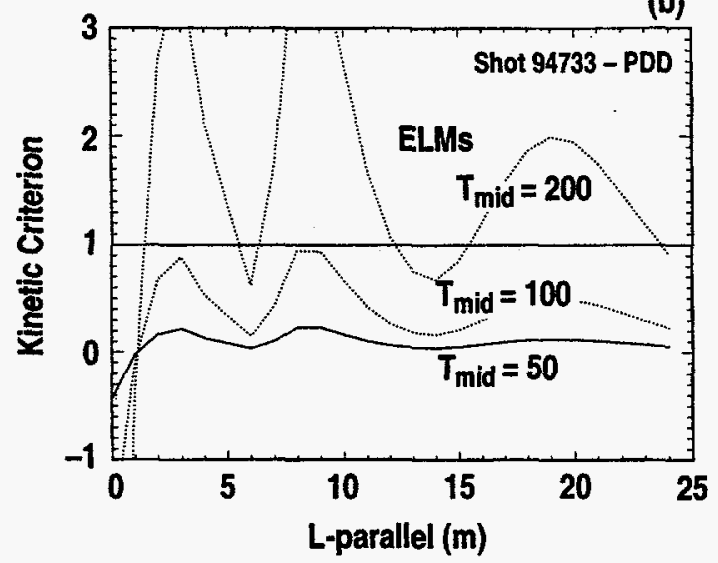

Fig. 4. These conditions near the separatrix $(1<$ normalized poloidal flux $(p s i-n)<1.002)$ along the field during $P D D(a)$ and the resulting kinetic criterion (b) are based on Thomson scattering data in the divertor.

$\mathrm{H}$-mode. The conditions do not violate the kinetic criterion calculated from the data because the density drop near the target occurs in a region where the temperature gradient is low. The population of fast particles in this case calculated from the distribution function (Fig. 5 ) is $0.2 \%$ of the background density. The fast electrons are thermalized by the higher density region below the X-point. Electrons should penetrate to the target during ELMs however.

Experimentally, we observe in this partially detached case, the $T_{e}$ measurements agree within $30 \%$. We observe large fluctuations in the Langmuir probe measurements (Fig. 6). ELMs at $200 \mathrm{~Hz}$ are indicated by the inner wall $\mathrm{D}_{\alpha}$ signal. Fluctuations dominate conditions here and make fitting probe characteristics difficult. By restricting the voltage range to near the floating potential and thus the time window over which the fit is performed, a much higher percentage of fits and more probe $T_{e}$ measurements at $2 \mathrm{eV}$ can be obtained. We still observe some fraction of the fits at higher $\mathrm{T}_{\mathrm{e}}$ which are associated with ELMs or ELM induced fluctuations. 


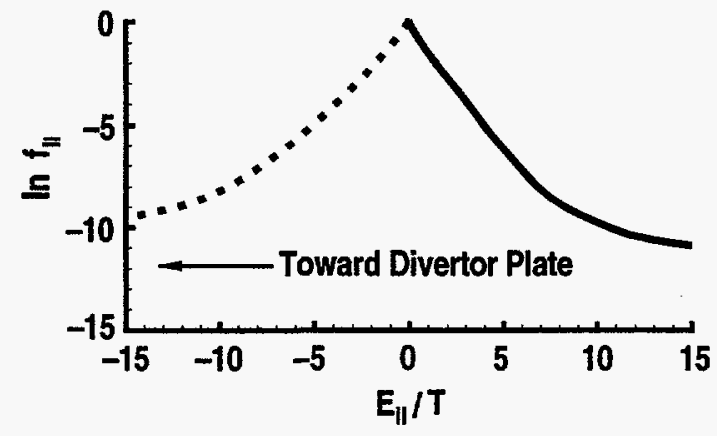

Fig. 5. The distribution function just above the target plate and calculated from the Fokker Planck code for the PDD mode is shown as a function of the normalized energy distribution of the bulk population of electrons.

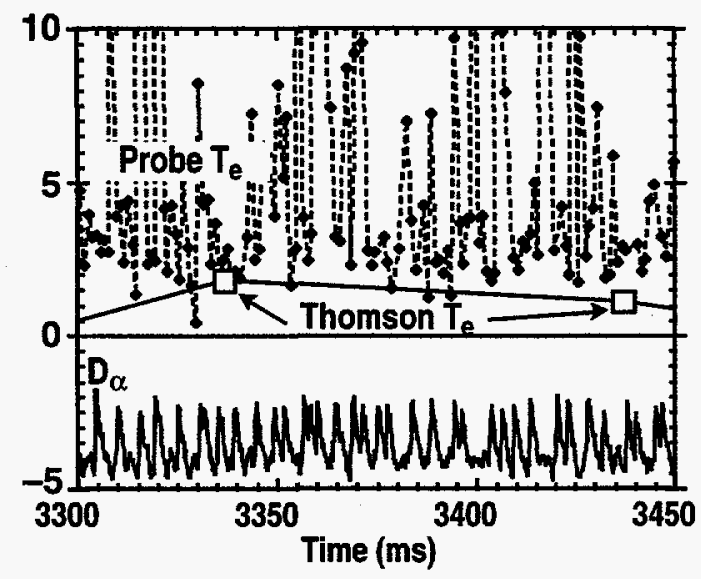

Fig. 6. This figure shows the $T_{e}$ comparison in PDD as a function of time. The fluctuations are readily apparent and are attributed to fast electrons penetrating the detached plasma during ELMs. 


\section{DISCUSSION}

\subsection{ELMING H-MODE}

The $T_{e}$ agreement between the Thomson and probe measurements is consistent with the kinetic criterion being less than one. The $\mathrm{T}_{\mathrm{e}}$ gradient and local density keep kinetic effects from affecting the steady state measurements. In this high recycling regime, the rise in density below the X-point increases the collisionality near the target and causes the temperature to drop more slowly as more neutrals are encountered and ionized by electrons flowing toward the target along the field.

The probe $T_{e}$ error is limited by the lower temperature gradient. There is only a factor of three between the target and the midplane and the electrons out on the tail at $3 \mathrm{kT}$ are both reduced in number and are many mean-free-paths away from the target. For a $2 \mathrm{~m}$ mean free path typical for these energies and densities, the divertor is about 5 mean-free-paths away. The population of these $3 \mathrm{kT}$ electrons would be reduced to $1 \%$ of the midplane density and another factor of 3 when compared to the higher target plate density.

The code calculations indicate that the distribution function in the divertor is not truly characterized by two Maxwellians at two different temperatures. The calculated distribution functions in Figs. 2 and 3 are a continuous evolution from the bulk temperature to an enhanced tail. The degree of enhancement varies along the field line depending on conditions. The population of electrons at $5 \mathrm{kT}$ at the divertor plate is about 2-3 orders of magnitude reduced from the normal Maxwellian levels. Probes normally make measurements around the floating potential of $3 \mathrm{kT}$ of the target plate condition.

When an ELM occurs, we expect that the kinetic criterion is violated. The criterion evaluated in Fig. 1 is almost exceeded for steady state conditions. The midplane temperature is observed to increase by $50 \%-100 \%$ percent during an ELM and this causes the criterion to be exceeded on the our averaged flux tube near the separatrix. Further out in the scrape-off layer, the temperature during an ELM can be as much as five times higher than between ELMs. The first probe fit during or just after an ELM is when the fast electrons would arrive and it is often rejected because the ion flux that follows saturates the $4 \mathrm{~A}$ current available from the power supply. During the ELM recovery phase after the fast electrons and ions have arrived at the target plate, the temperature is often observed to drop when the ions recycle as neutrals and get reionized The target plate probes can measure during these conditions. 


\section{2. $P D D$}

Since the improvements to the probe system to optimize low temperature measurements, the $\mathrm{T}_{\mathrm{e}}$ comparison with Thomson scattering is normally within $1.5 \mathrm{eV}$. The large fluctuations during PDD make it difficult to compare the measurements. Any disturbance during the sweep tends to raise the fitter temperature. The ELM frequency increases during PDD and results in disturbances on almost every voltage sweep. By reducing the fitter time window, the fitter is less likely to be disturbed (Fig. 6).

The higher $T_{e}$ points seen by the probe are due to fluctuations in the divertor. These fluctuations, probably driven by $\mathrm{T}_{\mathrm{e}}$ excursions (ELMS, turbulence) at the midplane, may also be associated with some divertor instability. Future correlation measurements will shed more light on this situation. From the probability distribution of the probe $\mathrm{T}_{\mathrm{e}}$ measurements shown in Fig. 7, one can see that the measurements are peaked around $2 \mathrm{eV}$ with a skewed rather than a Gaussian distribution. Averaging this type of distribution in the normal way will give a higher result than the most probable value. We note that the most probable values for the probes is $2.5 \mathrm{eV}$ and for Thomson scattering is about $1 \mathrm{eV}$. This difference may not be significant considering the difficult comparison for this case and is 2.5 times less than previous kinetic estimates.

\subsection{TURBULENCE}

High frequency fluctuations can effectively increase the average electron temperature resulting from fitting $\mathrm{I} / \mathrm{V}$ characteristics due to changes in both the floating potential and density during the sweep. Intermittent bursts of high energy electrons are the most probable cause of these fluctuations on the $V_{f}$ signals and may reflect fluctuations in the upstream distribution function near the separatrix. At low $T_{e}$, floating potential fluctuations result in large current fluctuations in the nonlinear region of the $I / V$ characteristic where $T_{e}$ is determined. Simulations of fluctuations on the ideal characteristic can simulate the experimental data and when input to the three parameter exponential fitter, which fits up to the floating potential, can result in $T_{e}$ measurements which are higher than the $T_{e}$ used to generate the ideal initial $I / V$ curve. The outer portion of the radial profile, where the signal level is low, has a lower signal /noise ratio and is more sensitive to these fluctuations. 

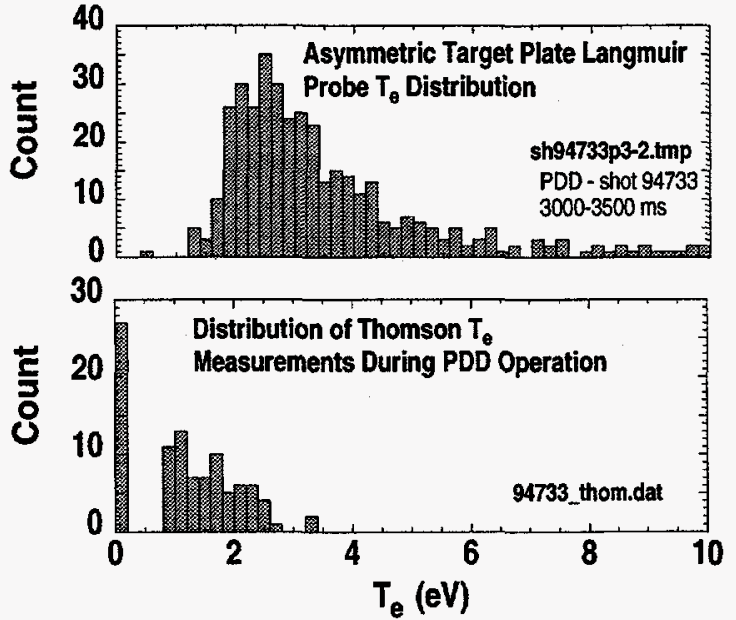

Fig. 7. The probability distributions of the probe $T_{e}$ and Thomson scattering measurements are show for the PDD plasma. Averaging this type of skewed distribution raises the mean value. The Thomson points at zero mean no measurement was possible for that laser pulse. 
14 


\section{CONCLUSIONS}

We have found that for two specific conditions in DIII-D, the code calculation of the fast electron tail of the distribution functions based on experimental data along a field line in the divertor yields $0.3 \%$ fast electrons for ELMing $\mathrm{H}-$ mode and $0.2 \%$ for PDD. This is consistent with the $\mathrm{T}_{\mathrm{e}}$ comparison. The higher density found in the divertor during these discharges and the $\mathrm{T}_{\text {mid }} / \mathrm{T}_{\mathrm{div}}$ ratios were consistent with estimates [2] of probe $\mathrm{T}_{\mathrm{e}}$ measurements of the bulk of the distribution. The high divertor density seems to dominate the temperature gradient in the divertor and absorb the fast tail electrons except during ELMs. We attribute the $\mathrm{T}_{\mathrm{e}}$ spikes on our probe measurements to fast electrons launched during ELMs which is consistent with our criterion estimates.

We have become more aware that fast electrons launched upstream during ELMs, while occupying only about two percent of the discharge time, account for a much larger fraction of the heat flux. The study of kinetic effects is a particularly rich area for physics studies of the divertor and scrape-off layer and has potential benefits for understanding the radiation and power flow in the divertor. Although these particles may not all make it to the target plate, they are likely playing a role in the divertor physics upstream. 


\section{REFERENCES}

[1] K. Kupfer, et al., Phys Plasmas 3 (1996) 3644.

[2] P. Stangeby, Plasma Phys. Contrl. Fusion 37 (1995) 1031

[3] A.W. Leonard, Phys. Rev. Lett. 78 (1997) 4769.

[4] T.N. Carlstrom, Rev. Sci. Instrum. 68 (1997) 1195.

[5] J.G. Watkins, et al., J. Nucl. Mater. 241-243 (1997) 645.

[6] K. Gunther, et al., J. Nucl. Mater. 176-177 (1990) 236.

[7] R.D. Monk, et al., J. Nucl. Mater. 241-243 (1997) 396.

[8] O. Batishchev, Phys. Plasmas 4 (1997) 1672.

[9] J. Lingertat, et al., J. Nucl. Mater. 220-222 (1995) 198. 


\section{ACKNOWLEDGMENTS}

Work supported by U.S. Department of Energy under Contract Nos. DE-AC03-89ER51114, DE-AC04-95AL85000, W-7405-ENG-48, and Grants DE-FG02-97ER54392, DE-FG0291ER54109, DE-FG03-95ER54294. 